\title{
The Application of Brain Functional Magnetic Resonance Imaging in Bilingual Teaching
}

\author{
Juan Lu and Lin Zhu
}

${ }^{1}$ School of Foreign Studies, Shaanxi University of Technology, Hanzhong 723000, Shaanxi, China;

${ }^{2}$ Department of Neurosurgery, 3201 Hospital, Zhonghang Industry, Hanzhong 723000, Shaanxi, China.

a672241343 @ qq.com, b504524541 @ qq.com

\begin{abstract}
With the rapid development of magnetic resonance imaging technology, the study on language functional imaging of bilingual people is deepening in the aspects of depth, breadth and precision. Development imaging more clearly high field strength magnetic resonance imaging system, ultra-fast imaging software and data post-processing software, making the secret of human language was revealed step by step. This paper focused on the application of magnetic resonance in bilingual teaching. It summarized the main findings and achievements of magnetic resonance technology in bilingual teaching. Based on the existing research, this paper further discussed the development trend of magnetic resonance in bilingual teaching. In the future of magnetic resonance technology under the linguistic research to provide research base and learn from experience.
\end{abstract}

Keywords: Magnetic resonance imaging; Bilingual teaching; Language functional area; Bilingual conversion mechanism

\section{脑功能磁共振成像技术在双语教学中的应用}

\author{
陆娟 ${ }^{1,}$ a , 朱林 ${ }^{2,}$ b \\ ${ }^{1}$ 陕西理工大学 外国语学院 陕西 汉中 723000 ; \\ 中航工业三二零一医院 神经外科陕西汉中 723000 .
}

摘要: 随着磁共振功能成像技术的快速发展, 对双语者大脑语言功能成像方面的研究在深度、广度 及精确度方面不断 深入。成像更加清晰的高场强磁共振成像系统的出现, 超快速成像软件及数据后处理软件的开发, 使得人类语言的秘密一步 一步被揭示。本课题着重研究磁共振在双语教学中的应用, 综述磁共振技术应用于双语教学中国内外的主要研究发现和成果, 在已有的研究基础上进一步探讨磁共振应用于双语教学的发展趋势, 旨在为未来的磁共振技术下的语言学研究提供研究基础 和借鉴经验。

关键词: 磁共振成像技术; 双语教学; 语言功能区; 双语转换机制

引言

语言作为人脑特有的功能, 吸引了无数科学家的研究热情。研究中英双语者脑功能并对其进行评估, 有利于揭示人脑的奥秘并造福于人类, 同时对双语教学与科研亦有着重要的科学意义。在磁共振功能成像 技术诞生之前, 由于技术条件的限制, 仅能做一些简单的问卷调查和量表分析, 既不能做动物实验, 如免 疫生化或组织病理学等方面研究, 也不能对受试者进行一些有创的或伤害性研究。20 世纪 90 年代初功 能磁共振的出现是神经影像学的重大发展, 它可以无创、实时地再现人类思维活动, 因而为当代最热门的 脑科学及人类语言学研究注入了新的活力。功能磁共振成像是一种安全的无创伤性的检查方法, 在执行语 言任务时, 通过测量局部神经活动导致的该区域血氧饱和度水平改变引起的磁场信号变化, 揭示参与语言 
处理的相关脑区。通过图像分析和处理。可以在人脑的结构图像上直观地展现有关功能区。现阶段, 语言 磁共振功能成像研究内容主要包括: (1). 正常人不同语言刺激模式时的大脑皮层定位研究; (2). 语言偏 侧指数 FMRI 研究; (3). 双语者不同语种语言区研究。每一方面研究又分为词语水平与句子水平研究, 而 双语者大脑语言区的研究为目前热点。

\section{1 脑功能磁共振成像技术在双语教学中的应用研究}

国外学者 Seiji Ogawa 首次将磁共振功能成像技术应用于人脑功能的研究, 从此人类对于语言的探索 有了突飞猛进的突破。1992 年, Sakuri 等把磁共振功能成像技术应用到日语这种特殊构成组合的语言中, 探测大脑皮层的语言功能区。证据表明阅读日语的受试者同阅读英语的受试者相比, 其大脑激活区域有本 质的不同。1994 年, Klein 等学者用电子发射断层成像技术研究发现, 用母语和第二语言命名图片时, 都 有左侧额叶的激活, 这说明语言加工处理的不同阶段, 如语音和语义、句法和句义、语调和语气等既有共 同的脑区激活模式, 也有其独特性, 进一步显示了人类语言现象的复杂性。从此以后, 双语研究成为一个 引人关注的课题。1998 年, Nakada 等选择了 10 名掌握日语及英语的双语者, 对其进行句子水平的阅读研 究。利用高场强磁共振功能成像, 在清晰的解剖定位的优势下, 实验结果表明: 所有日本人在阅读日语时, 都发现有左题下回腹后侧区的活动明显增加, 而在阅读英语时无此现象发生。2000 年, Hernades 等利用 FMRI 研究运用单语种和语种转换两种条件下, 命名图片时的相关脑功能。结果发现: 凡是能够导致大脑处 理耗能产生的语言转换, 都会引起额叶前部背侧区的活动曾加。Pilai 对英语和西班牙语双语者进行语义 和语音任务测试, 结果发现加工处理第二语言的神经活动过程有别于母语。Perani 做实验要求双语者听一 篇分别用母语和第二语言念出的文章, 结果发现受试者左侧大脑的母语和第二语言激活区明显不同。Kim 等则对汉英双语者进行拼写形式和词义判断任务, 结果提示第二外语 (英语) 较汉语更多的利用了右大脑半 球功能。

国内运用磁共振功能成像研究语言起步相对较晚, 但研究更加深入。2006 年, 李恩中等运用磁共振功 能成像对语言与音乐刺激下脑功能活动进行研究, 结果证实: 语言刺激时, 主要为左大脑半球的脑区激活, 右侧半球部分脑区也可被激活; 而在音乐刺激时主要为右侧半球激活, 说明左、右大脑半球在语言和音乐 加工时存在分工优势性, 但同时两半球间及半球内各脑区有相应协同作用。2007 年, 韩立新等运用磁共振 功能成像进行汉语同义字和同音字测试, 结果发现两种语言任务均能明显激活 Broca 区、Wernicke 区、双 侧纹外视区及双侧题叶腹侧皮质两者所激发的脑功能区之间无显著性差异，且可重复性好。2009 年, 唐 一源等采用读词名的方法研究汉字词及假词视觉识别时的脑功能偏侧化现象, 结果表明, 汉字词激活左额 下区、右题叶及右枕叶, 而假词除额区外表现广泛激活, 提示除左脑半球与汉字词加工密切相关外, 右脑 半球在汉字词加工中有一定参与, 但确切机制有待进一步研究。2010 年, 彭聑龄等采用磁共振功能成像研 究汉字单词音、义加工的脑机制, 结果表明在语义任务中与语音有关的脑区得到激活, 而在语音任务中与 语义有关的脑区没有激活。该实验也支持汉语单字词加工的非知觉观点及 “普遍语音” 的观点。2011 年, 刘树永博士用两种语言刺激模式对照研究语言区显示效果, 结果发现不同的语言刺激任务根据任务的性质 和难度不同激活脑区及强度不同, 语言任务的完成需多个脑区的协同作业。以上研究表明, 语言区的激活 并非仅依赖经典的语言皮层区，而是由许多大脑皮质及皮质下核团构成的巨大神经网络进行协调工作产生 的, 语言的产生过程和认知过程密切相关。所有试验都发现受试者在受到母语刺激和第二外语刺激时大脑 
的语言激活区域明显不同, 遗憾的是没有进一步研究双语者在进行语言加工时的双语转换机制, 本课题将 在前人的研究基础上弥补这一空白。

\section{2 发展趋势}

通过比较人脑语言磁共振功能成像差异，观察英汉双语者在进行语义加工时双侧大脑半球激活区的异 同, 了解相应皮质活动中枢的分布及双侧大脑半球的工作模式, 研究双语者语言转换机制, 研究人脑语言 功能及双语脑可塑性, 为双语者学习和教师教学提供理论依据并指导教学工作。随着科学技术的进步及医 学的发展, 功能神经影像学日趋成熟, 尤其是基于血氧水平依赖的磁共振功能成像（FMRI）技术在不断提 高, 它能结合脑形态学和功能学研究方法, 被广泛应用于神经语言学研究中。近期的研究结果不仅证实了 既往研究的经典理论, 而且进一步发展了语言区功能定位, 为阐释语言产生过程及双语转换过程提供依据, 为双语者学习和教师教学提供理论依据并指导教学工作。人脑的功能异常复杂, 语言磁共振功能成像在 21 世纪的研究还有很多问题, 比如母语和第二语言的生成和理解是否由同一个脑神经系统执行; 比如现在大 部分学习者都是以视觉型为主, 如何让听觉编码在语言学习中起主导作用等, 还需语言学界、医学界、心 理学界等多学科研究者的共同努力。

人脑磁共振功能成像作为一种新的成像技术，在探索人类神经活动包括思维的生理机制方面有很大的潜 力和广泛的前景。它可以再现人类的思维活动, 为人类最热门的脑科学及人类语言学注入了新的活力。成 像更加清晰的高场强磁共振成像系统的出现, 超快速成像软件及数据后处理软件的开发, 使磁共振研究条 件日渐优越。利用磁共振功能成像技术可以研究不同脑区之间的功能相关性 (functional connectivity), 脑部在静息状态下自发的低频活动的同步化现象广泛存在于听觉、视觉和工作记忆系统内, FMRI 与弥散张 量成像 (DTI)、脑磁图 (MEG)、经颖磁刺激（TMS）等技术相结合，可得到更多的脑功能活动信息。本实 验的研究发现不仅可以应用于人脑语言功能研究及双语脑可塑性研究, 为双语者学习和教师教学提供理论 依据并指导教学工作, 还可以应用于失语症语言恢复、癫㾋患者语言恢复, 脑卒中患者语言恢复及乑内受 伤患者语言恢复。

神经影像学方法现已越来越多地应用于科研和临床中。功能影像学的应用不仅能够反映双语者脑形态 学, 而且能够反映功能学的变化, 为研究语言在大脑的功能定位提供了技术和方法, 为深入了解语言功能 的神经基础, 进一步揭示失读和失写等语言功能障碍的病理生理机制, 提供了有力的技术手段。同时, 双 语者脑功能活动研究对双语学习和教学也有重要意义。

\section{3 结语}

目前国内外常用的研究中英文双语者脑功能的方法是 FMRI 和 ERP。由于这些研究手段的缺陷与限制, 不同的研究手段可能会影响其研究结岥。因而, 综合使用不同的研究方法是隧前研究中英文双语誊脑功能 最有效的研究方式。当然还有很多因素会影响中苂双语者脑功能的研究, 包括第二语言的熟练程度、获得 第二语言的年龄、双语者习得第二语言的方式等。除了以上双语者本身的因素, 两种语言的相似性、具体 的词汇类型等也是影响双语袭征与加工的重要因素。因此, 配合使用各种神经心理学量表和行力学实验也 会起到重要的作用。榴信随着科学技术懿发展, 对双语脑功能研究与评估方法会日趋完善, 人类语言的秘 密终究将会得以揭示。 


\section{4 致谢}

基金项目：本论文为陕西省教育厅基金项目的部分研究成果，陕西省教育厅基金项目的项目名称为： 新丝绸之路经济带建设背景下的陕西复合型外语人才培养研究 (15JK1130)。

\section{Acknowledgement}

Fund Project: This paper is part of the research results of Shaanxi Province Education Department Fund Project. The title of Shaanxi Province Education department Fund Project is: Research on the Cultivation of Interdisciplinary Foreign Language talents in Shaanxi province under the background of The New Silk Road Economic Zone construction (15JK1130).

\section{参考文献:}

[1] Abutalebi J, Annoni JM, Zimine I, et a1. Language control and lexical competition in bilinguals: An event-related FMRI study [J]. Cerebral Cortex, 2008, (7): 149-152.

[2] Clements-stephens AM, Materek AD, Eason SH, et a1. Neural circuitry associated with two different approaches to novel word learning [J]. Dev Cogn-Neurosci, 2012, (6): 99-113.

[3] Gartus A, Foki T, Geissler A, et a1. Improvement of clinical language localization with all overt semantic and syntactic language functional MR imaging paradigm [J]. AJNR Am J Neuroradiol, 2009, (10):1977-1985.

[4] Jones SE, Mahmoud SY, Phillips MD. Apractical clinical method to quantify language lateralization in FMRI using whole-brain analysis [J]. Neuroimage, 2011, (7): 2938-2949. 